\title{
Silencing of ILK attenuates the abnormal proliferation and migration of human Tenon's capsule fibroblasts induced by TGF-ß2
}

\author{
YAO XING $^{1}$, LIJUN CUI ${ }^{2}$ and QIANYAN KANG ${ }^{2}$ \\ ${ }^{1}$ Medical School of Xi'an Jiaotong University; ${ }^{2}$ Department of Ophthalmology, The First Affiliated Hospital \\ of Medical School of Xi'an Jiaotong University, Xi'an, Shaanxi 710061, P.R. China
}

Received November 2, 2015; Accepted June 1, 2016

DOI: $10.3892 / \mathrm{ijmm} .2016 .2644$

\begin{abstract}
The cytokine, transforming growth factor- $\beta$ (TGF- $\beta$ ), plays a key role in wound healing and tissue repair. Integrin-linked kinase (ILK) is a downstream factor of the TGF- $\beta$ signaling pathway. Research on ILK has mainly focused on its role in the invasion and metastasis of cancer cells. However, little has been reported on the effects of ILK in human Tenon's capsule fibroblasts (HTFs). In this study, we investigated the role of ILK in the proliferation and migration of HTFs exposed to TGF- $\beta 2$. A lentiviral vector targeting ILK was screened from three candidates and the experimental result indicated that RNA interference can be used to inhibit ILK expression at both the mRNA and protein level. Reverse transcription-quantitative polymerase chain reaction (RT-qPCR) was used to assess ILK mRNA expression. Cell proliferation was quantified by MTT assay and cell cycle progression was detected by flow cytometric analysis. Migration was measured by wound healing assay. It was observed that the silencing of ILK suppressed the TGF- $\beta 2$-induced proliferation of HTFs and led to G1 phase cell cycle arrest and the significant downregulation of cyclin D1 expression. The migration ability of the HTFs decreased following the silencing of ILK, while the downregulation of $\alpha$-smooth muscle actin expression and the upregulation of E-cadherin expression were observed. The findings of our study indicate that the silencing of ILK attenuates the abnormal proliferation and migration of HTFs induced by TGF- $\beta 2$, which reveals the therapeutic potential of ILK inhibition in the prevention of scarring following glaucoma filtration surgery.
\end{abstract}

\section{Introduction}

Glaucoma is the second leading cause of blindness worldwide, disproportionately affecting women and Asians (1). It is a

Correspondence to: Dr Lijun Cui, Department of Ophthalmology, The First Affiliated Hospital, Medical School of Xi'an Jiaotong University, 76 Yanta West Road, Xi'an, Shaanxi 710061, P.R. China E-mail: grace_cuicui@163.com

Key words: transforming growth factor $\beta 2$, human Tenon's capsule fibroblasts, scarring following glaucoma filtration surgery, extracellular matrix, fibroblasts, $\alpha$-smooth muscle actin, cyclin D1, E-cadherin neurodegenerative disease characterized by elevated intraocular pressure (IOP), which causes damage to visual function and optic nerves. Glaucoma filtration surgery is the most frequent technique applied to reduce IOP in cases of uncontrolled glaucoma. However, the long-term success is often impaired by the post-operative wound healing process. Fibroblasts from Tenon's capsule are involved in the process that finally leads to the obstruction of the created fistula and subconjunctival filtration area (2). In recent years, 5-fluorouracil (5-FU) and mitomycin C (MMC) have been found to reduce scar formation; however, their applications are limited by severe side-effects (3). Therefore, great attention has been paid to the therapy of post-operative scar formation and it is exigent to find safe and effective therapeutic strategies.

Transforming growth factor- $\beta$ (TGF- $\beta$ ) is believed to be a pivotal mediator, driving both normal wound healing and tissue fibrosis. There are three TGF- $\beta$ isoforms in humans: TGF- $\beta 1$, TGF- $\beta 2$ and TGF- $\beta 3$, of which TGF- $\beta 2$ has been found to be the predominant isoform in ocular scarring-related diseases, such as conjunctival scarring and proliferative vitreoretinopathy (4). Protein and mRNA of TGF- $\beta$ receptors type I, II and III are expressed in cultured human Tenon's capsule fibroblasts (HTFs). A significant increase in proliferation has been detected following exogenous stimulation with TGF- $\beta 1$ and TGF- $\beta 2$ (5).

To reduce the abnormal proliferation of fibroblasts, previous studies have mostly focused on the inhibition of Smads $(6,7)$. However, Smads are located upstream of the TGF- $\beta$ signaling pathway which contains multitudinous target genes (8). The complete inhibition of Smads may lead to poor wound healing $(9,10)$. Therefore, it has become a critical issue to search for safer and more effective inhibition targets downstream of the TGF- $\beta$ signaling pathway. Recent studies have indicated that integrin-linked kinase (ILK) is a serine/threonine protein kinase located in focal adhesions, which regulates cell growth, proliferation, survival, differentiation, migration and invasion $(11,12)$. In earlier experiments, ILK was shown to play an important role in mediating epithelial-mesenchymal transition (EMT) induced by TGF- $\beta 1$. TGF- $\beta 1$ induced ILK expression in renal tubule epithelial cells in a dose-dependent manner, which was dependent on intracellular Smad signaling. ILK also induced matrix metalloproteinase-2 (MMP-2) expression and promoted cell migration and invasion in Matrigel (13). Simultaneously, ILK-deficient fibroblasts treated with TGF- $\beta 1$ have been shown to exhibit decreased Smad 2 and 3 phosphorylation, accompa- 
nied by the impaired transcriptional activation of Smad targets, such as $\alpha$-smooth muscle actin ( $\alpha$-SMA). ILK-deficient fibroblasts also exhibited abnormalities in the actin cytoskeleton, indicating a severe impairment in their capacity to differentiate into myofibroblasts (14). On the other hand, in the study of tumors, ILK overexpression, the downregulation of E-cadherin and the activation of Akt have been observed in primary colon carcinoma (15). The overexpression of ILK has also been shown to promote glioma cell invasion and migration, and to downregulate E-cadherin (16). At the same time, the inhibition of ILK has been show to induce G1 phase cell cycle arrest and promote apoptosis in PTEN-negative prostate cancer cells (17). The levels of ILK expression correlate strongly with tumor invasion and cancer cell proliferation. Experiments on the effects of ILK in HTFs, however, have not been reported to date, at least to the best of our knowledge. ILK may meet the requirements for a novel therapeutic target and may thus be an important factor in scarring following glaucoma filtration surgery. RNA interference (RNAi) has been proven to be one of the most potent, robust, and easy-to-use tools for the inhibition of gene expression. Short interfering RNAs (siRNAs) are now used routinely to assess the role of selected genes in cell-based assays (18).

In this study, HTFs were cultured in vitro following the delivery of ILK-targeted siRNA by lentiviral vectors. The proliferation and migration of HTFs induced by TGF- $\beta 2$ were investigated. The effects of the silencing of ILK in vitro on cell cycle progression, and on $\alpha$-SMA and E-cadherin expression were examined simultaneously. ILK was shown to have the potential to influence cell-signaling events linked to fibroblast activation and differentiation, which provides the basis for further study.

\section{Materials and methods}

Primary cell culture. HTFs were obtained from excised Tenon's capsule specimens during glaucoma surgery. Written informed consent was obtained prior to operative excision and this study was approved by the Research Ethics Committee of Xi'an Jiaotong University, Xi'an, China. Tenon's capsule tissues $(1 \times 5 \times 3 \mathrm{~mm})$ were resected during surgery and placed in a 60-mm culture dish containing Dulbecco's modified Eagle's medium (DMEM) supplemented with $15 \%$ fetal bovine serum, $100 \mathrm{U} / \mathrm{ml}$ penicillin and streptomycin (HyClone, Logan, UT, USA). Tissues were cut into small sections $\left(1 \mathrm{~mm}^{3}\right)$ and were then incubated in $25 \mathrm{~cm}^{2}$ culture bottles at $37^{\circ} \mathrm{C}$ in a $5 \% \mathrm{CO}_{2}$ environment. The cells were allowed to migrate from the explant tissue and observed under an inverted phase contrast microscope (COIC IBE1000; Chongqing COIC Industrial Co., Ltd, Chongqing, China). The medium was changed every 2-3 days and the cells were allowed to reach $80 \%$ confluence. Subsequently, the cells were disaggregated with $0.25 \%$ trypsin and $0.02 \%$ EDTA at $37^{\circ} \mathrm{C}$ for $3 \mathrm{~min}$ and were passaged every 3-5 days. Cells that maintained their proliferative potential and acquired a fibroblast-like elongated morphology between the third and fifth passage were used in this study (data not shown). To examine the purity of HTF cultures, immunofluorescence was staining was performed with vimentin and keratin. The stained cells were observed under an immunofluorescence microscope (Leica DMI3000B; Leica Microsystems GmbH, Wetzlar, Germany).
Construction of ILK-siRNA lentiviral vectors. The lentivirus expressing siRNA targeting ILK (GeneChem, Shanghai, China) was constructed to inhibit ILK gene expression in order to examine the effects of ILK on the proliferation and migration of HTFs. Green fluorescent protein (GFP) was used as a reporter gene transferred into the HTFs and the siRNA (5'-GCC GTAGTGTAATGATTGA-3') sequence for ILK was designed using the manufacturer's RNAi designer program, and the negative control construct (control siRNA) was created using a scrambled sequence (5'-TTCTCCGAACGTGTCACGT-3'). DNA oligos were chemically synthesized (GeneChem), annealed and inserted into the expression vector by double digestion with AgeI and EcoRI (New England Biolabs, Ipswich, MA, USA), and ligated with T4 DNA ligase (Takara Biotechnology Co., Ltd., Dalian, China) in accordance with the manufacturer's instructions. The ligation was transformed into competent $E$. coli cells and then confirmed by restriction enzyme analysis and DNA sequencing. These quences were then cloned into pGCSIL-GFP to generate lentiviral vectors. The expression vectors and package vectors were then transfected into 293T cells (ATCC, Manassas, VA, USA) using Lipofectamine 2000 (Invitrogen, Carlsbad, CA, USA). Following $48 \mathrm{~h}$ of culture, the supernatants containing the lentiviruses, such as ILK-siRNA-LV and NC-GFP-LV (negative control) were harvested. Purification was then performed using ultracentrifugation and the lentiviral titer was determined.

Lentivirus-mediated RNAi silencing of ILK. The cells $\left(5 \times 10^{4}\right.$ cells/well) were seeded in 6 -well cell culture plates the day before transfection. The lentivirus $\left(1 \times 10^{8} \mathrm{TU} / \mathrm{ml}, 50 \mu \mathrm{l}\right)$ was mixed with the cells $\left(1 \times 10^{5}\right.$ cells/well) in serum-free medium, and the medium was replaced by fresh cell medium containing $15 \% \mathrm{FBS}$ at $24 \mathrm{~h}$ post-transfection. The positive cells were selected by puromycin $(2.5 \mu \mathrm{g} / \mathrm{ml}$; Sigma-Aldrich, St. Louis, MO, USA), and then further cultured for 5 days. The cells were divided into 3 groups as follows: i) the normal control group (no LV transfection); ii) the negative control LV-transfected group; and iii) the group transfected with ILK-siRNA-LV. The cells were observed under a fluorescence microscope (Leica DMI3000B; Leica Microsystems GmbH).

Reverse transcription-quantitative polymerase chain reaction $(R T-q P C R)$. Total RNA was extracted from the cells using an RNA extraction kit (Fastagen Biotech., Shanghai, China) and reverse transcribed into complementary DNA (cDNA) using the PrimeScript 1st Strand cDNA Synthesis kit (Takara) following the manufacturer's instructions. The quality of the RNA samples was controlled by measuring the absorbance at A260/280; absorptions between 1.8 and 2.1 indicate good quality. qPCR was used to evaluate the knockdown efficiency of ILK, using SYBR Premix Ex Taq ${ }^{\mathrm{TM}}$ II (Tli RNaseH Plus) following the manufacturer's instructions (Takara Biotechnology Co., Ltd.). The primers used for PCR were as follows: ILK forward, 5'-CCCAACACAAACACTTCTCTCCTG-3' and reverse, 5'-AAGCCTGAGGACTGTGGAGTGAT-3'; and GAPDH forward, 5'-GCACCGTCAAGGCTGAGAAC-3' and reverse, 5'-ATGGTGGTGAAGACGCCAGT-3'. The program was initially run for $30 \mathrm{sec}$ at $95^{\circ} \mathrm{C}$, followed by 40 cycles of $5 \mathrm{sec}$ at $95^{\circ} \mathrm{C}, 20 \mathrm{sec}$ at $60^{\circ} \mathrm{C}$ and $20 \mathrm{sec}$ at $72^{\circ} \mathrm{C}$. The qPCR analysis used a Bio-Rad IQ5 multicolor detection system 
(Bio-Rad Laboratories, Inc., Hercules, CA, USA). A comparative cycle threshold method was used to determine the relative quantification of RNA expression. Gene expression levels were normalized to the GAPDH endogenous control and fold changes were calculated using the $\Delta \Delta \mathrm{Ct}$ method. All PCR reactions were performed at least in triplicate.

MTT assay. The HTFs in the 3 groups were seeded in 96-well cell culture plates. The cells were then cultured for $48 \mathrm{~h}$ in the presence or absence of TGF- $\beta 2$ (3 ng/ml). MTT $(20 \mu \mathrm{l})(5 \mathrm{mg} /$ $\mathrm{ml}$ in PBS) was added to each well, and the cells were then incubated at $37^{\circ} \mathrm{C}$ for $4 \mathrm{~h}$. MTT solution was discarded by gently inverting the plates, and the wells were filled with $200 \mu \mathrm{l}$ DMSO. After the plates were shaken vigorously for $20 \mathrm{~min}$, the absorbance of each well was read using a plate reader (Epoch $^{\mathrm{TM}}$; Bio-Tek Instruments Inc., Winooski, VT, USA) at $490 \mathrm{~nm}$. Each data point was obtained as an average of five values from five wells.

Wound healing assay. The HTFs were seeded in 6-well cell culture plates at a concentration of $5 \times 10^{5}$ cells/well and allowed to grow to $80 \%$ confluence. The cells were starved overnight and wounds were gently made in the center of the cell monolayer using a sterile $200 \mu \mathrm{l}$ pipette tip. To remove the cell debris, the wells were washed twice with PBS, and then incubated with or without TGF- $\beta 2$ ( $3 \mathrm{ng} / \mathrm{ml})$. Images of marked regions along the wound area were obtained using an inverted phase contrast microscope (Leica DMI3000B; Leica Microsystems GmbH) immediately after creating the wound. The width of the scratch was determined by images taken every $24 \mathrm{~h}$, employing Adobe Photoshop software.

Cell cycle analysis. The HTFs in the 3 groups were seeded in $25 \mathrm{~cm}^{2}$ culture bottles at a density of $1 \times 10^{6}$ cells/bottle. The cells were starved overnight and were cultured in the presence or absence of TGF- $\beta 2(3 \mathrm{ng} / \mathrm{ml})$. Following incubation at $37^{\circ} \mathrm{C}$ for $48 \mathrm{~h}$, the cells were collected and the cell suspension was added into $75 \%$ ice-cold ethanol and fixed overnight. The cells were then washed twice with PBS and re-suspended in $400 \mu \mathrm{l}$ of solution containing PI $(100 \mu \mathrm{g} / \mathrm{ml})$ and RNase $(0.1 \mathrm{mg} / \mathrm{ml})$ and then incubated in the dark for 20 to $40 \mathrm{~min}$. Finally, the cell cycle distribution was detected by flow cytometry, and the cells were analyzed using a FACSCalibur and FACSort CellQuest software (BD Biosciences, Franklin Lakes, NJ, USA).

Immunofluorescence staining. The cells $\left(1 \times 10^{5}\right.$ cells/well) were seeded on an $8 \times 8 \mathrm{~mm}$ cover slip in 24-well plates, starved overnight and then treated with $3 \mathrm{ng} / \mathrm{ml}$ TGF- $\beta 2$. Following $48 \mathrm{~h}$ of treatment, the cells were fixed with $4 \%$ paraformaldehyde for $20 \mathrm{~min}$ and washed 3 times with PBS. The cells were permeabilized in PBS with $0.3 \%$ Triton X-100 for $5 \mathrm{~min}$. The cells were then blocked with $5 \%$ normal goat serum for $1 \mathrm{~h}$. Primary rabbit anti-human $\alpha$-SMA antibody (1:500, ab5694) and rabbit anti-human E-cadherin antibody (1:1,000, ab53226) (both from Abcam, Cambridge, MA, USA) were incubated with the cells overnight at $4^{\circ} \mathrm{C}$. After being rinsed extensively, the cells were exposed to the fluorescent secondary antibody (goat anti-rabbit Cy3, 1:10,000, CW0114S; goat anti-rabbit FITC, 1:10,000, CW0159S; both from CWBIO, Beijing, China) in the dark for $30 \mathrm{~min}$. The coverslips were rinsed again with PBS
3 times and mounted with DAPI for 5 min, as a nuclear counterstain. Finally, the cells were observed under a fluorescence microscope (DP71; Olympus, Tokyo, Japan).

Western blot analysis. Proteins were separated using SDS-PAGE and transferred onto a PVDF membrane. The membranes were blocked in TBST containing 5\% skim milk at room temperature for $3 \mathrm{~h}$. Following this, the membrane was exposed to the primary antibodies (rabbit anti-human anti-cyclin D1 antibody, 1:1,000; ab134175), (anti-E-cadherin antibody, 1:1000), (anti-integrin-linked ILK antibody, 1:1000; ab52480), and (anti- $\alpha$-SMA antibody, 1:500) (all from Abcam) in a sealed bag overnight at $4^{\circ} \mathrm{C}$ and then washed 3 times for 15 min each in TBST. The membranes were then incubated with secondary antibody (goat polyclonal secondary antibody to rabbit $\lg$ G-HRP, ab6721), diluted at 1:10,0000 in TBST for $2 \mathrm{~h}$ at room temperature. The membranes were washed 3 times in TBST for $20 \mathrm{~min}$ each time. A Genshare ECL substrate solution (Genshare, Shaanxi, China) was placed on the membranes for $2 \mathrm{~min}$. Subsequently, the membranes were placed in a LAS-3000 FujiFilm intelligent dark box. The illuminated bands were detected and the image captured using Image reader LAS-3000 software. Western blot analysis was performed at least on 3 separate occasions.

Statistical analysis. All the data are presented as the means \pm standard deviation (SD). Statistical analyses were conducted using SPSS version 18.0 for Windows (SPSS, Inc., Chicago, IL, USA). The statistical comparisons were performed using a Student's t-test or ANOVA followed by planned comparisons of multiple conditions. A value of $\mathrm{P}<0.05$ was considered to indicate a statistically significant difference.

\section{Results}

Cell morphological observation and identification of HTFs by immunofluorescence staining. The cells migrated out from the explant tissue after 7 to 10 days of adherent culture. Microscopically, the cells exhibited a fusiform appearance with clear outlines, and the cellular plasma was abundant and bright, and the size uniform (Fig. 1A). The cells were passaged every 3-5 days. Following subculture, the cells had spread much more and were arranged in fasciculus or swirling patterns (Fig. 1B). There were no differences observed between the recovered cells and those before cryopreservation as regards morphology and growth characteristics. The results revealed that the cells were positive for the expression of vimentin, and the specific fluorescence could be observed within the cytoplasm (Fig. 1C and D). The cells were negative for the expression of keratin (Fig. 1E and $\mathrm{F}$ ).

RNAi can be used to inhibit ILK expression in HTFs. The cells were transfected with different levels of viral titer. When the multiplicity of infection (MOI) was 50, the transfection efficiency was the highest. The positive rate was $95 \%$ following selection with puromycin. The expression of GFP was positive in the ILK-siRNA-LV-transfected group (Fig. 2A and B) and in the negative control LV-transfected group (Fig. 2C and D) on the 4th day after transfection, but it was negative in the normal control group (Fig. 2E and F). The results of RT-qPCR revealed 

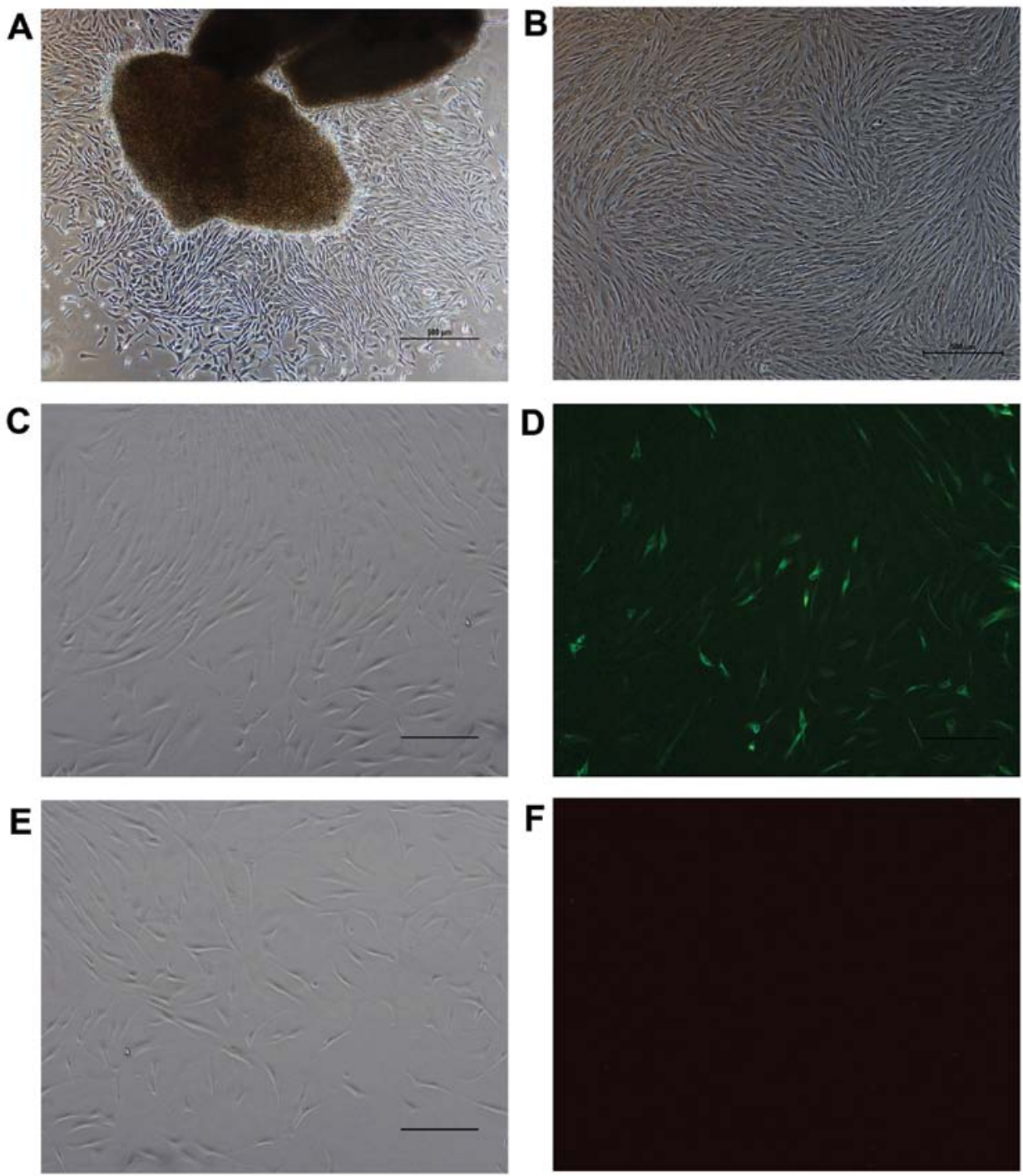

Figure 1. Cells migration from the explant tissue (x50 magnification). (A) Following subculture for 4 days, the cells were arranged in a fasciculus or swirling pattern. (B) Identification of human Tenon's capsule fibroblasts (HTFs) by immunofluorescence staining. Cells were stained for keratin and vimentin and observed under an immunofluorescence microscope. (C and D) Cells stained positive for vimentin; (E and F) cells stained negative for keratin. Scale bars, $500 \mu \mathrm{m}$.

that ILK mRNA expression was significantly decreased in the ILK-siRNA-LV-transfected group (Fig. 3).

Silencing of ILK attenuates the abnormal proliferation of HTFs induced by TGF- $\beta 2$. Based on cell culture model in vitro, MTT assay was used to examine the role of ILK in the proliferative activity of HTFs exposed to TGF- $\beta 2$. At concentrations of $3 \mathrm{ng} /$ $\mathrm{ml}$, TGF- $\beta 2$ induced an increase in proliferation in the lentivirustransfected group. However, we found that TGF- $\beta 2$ had no obvious promoting effect on the proliferation of HTFs in the normal control group. Furthermore, in the presence or absence of TGF- $\beta 2(3 \mathrm{ng} / \mathrm{ml})$, the cells transfected with ILK-siRNA-LV exhibited a lower proliferative activity (Fig. 4).

HTFs have a potent migratory ability; this migratory ability is decreased following the silencing of the ILK gene. Since we found that transfection with ILK-siRNA-LV suppressed the proliferation of HTFs induced by TGF- $\beta 2$, we examined the hypothesis that the silencing of the ILK gene has the potential to suppress the migration of HTFs. Wounds were made in the center of cell monolayers (Fig. 5A) and the migration distance in each group was similar at $24 \mathrm{~h}$ (Fig. 5B). The distance covered by cells in the ILK-siRNA-LV-transfected group (Fig. 5C, panels a and d) was shorter than that covered by the cells in the negative control LV-transfected group (Fig. 5C, panels $\mathrm{b}$ and e) and the normal control group (Fig. 5C, panels $\mathrm{c}$ and f) at $48 \mathrm{~h}$ (Fig. 5C). The wound had healed in the control group at $72 \mathrm{~h}$; however, there were still gaps observed in the ILK-siRNA-LV-transfected group (Fig. 5D). These data indicated that the migratory ability of the HTFs decreased after ILK gene silencing, although there was no significant differences observed between the same groups (transfected groups) of cells exposed to TGF- $\beta 2$ (Fig. 5 panels d-f) and those that were not exposed to TGF- $\beta 2$ (Fig. 5 panels a-c).

Silencing of ILK induces G1 phase cell cycle arrest in HTFs. The percentages of cells in the G1/G0 phase in the NC-GFPLV-transfected group and the normal control group exposed to TGF- $\beta 2$ decreased, while the percentages of cells in the $\mathrm{S}$ and $\mathrm{G} 2 / \mathrm{M}$ phases increased compared with the control cells 

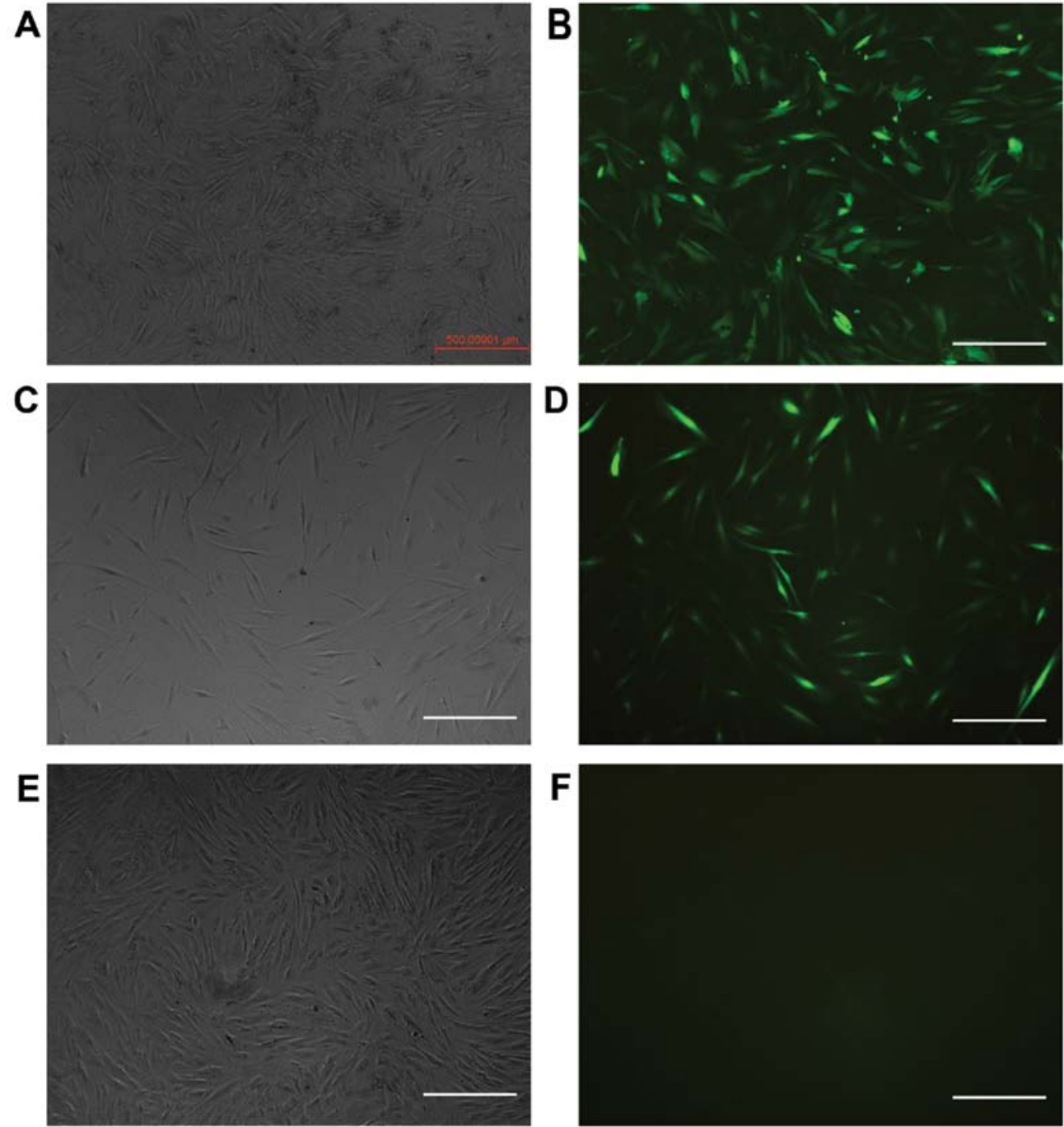

Figure 2. The cells were observed under a fluorescence microscope (x50 magnification) on the 4th day post-transfection. (A and B) Cells were positive for the expression of green fluorescent protein (GFP) in the integrin-linked kinase (ILK)-siRNA-LV-transfected group; (C and D) GFP expression was also positive in the negative control LV-transfected group. (E and F) GFP expression was negative in the normal control group. Scale bars, $500 \mu \mathrm{m}$.

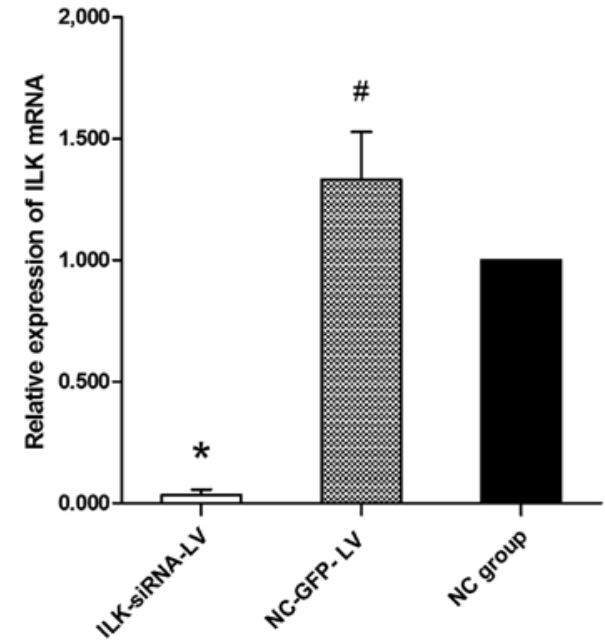

Figure 3. mRNA expression of integrin-linked kinase (ILK) by RT-qPCR. The expression of ILK was significantly decreased in the ILK-siRNA-LVtransfected group $\left({ }^{*} \mathrm{P}<0.05\right)$. A statistically significant difference was also observed between the transfected groups and the negative control (untransfected) group $\left({ }^{\#} \mathrm{P}<0.05\right)$.

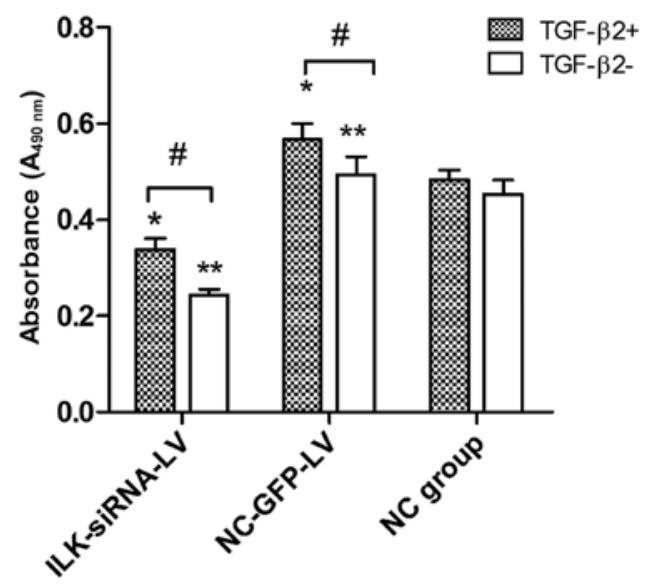

Figure 4. The results of MTT assay revealed that exposure to transforming growth factor- $\beta 2$ (TGF- $\beta 2)(3 \mathrm{ng} / \mathrm{ml})$ enhanced the proliferative ability of the cultured human Tenon's capsule fibroblasts (HTFs) in the integrin-linked kinase (ILK)-siRNA-LV-transfected group and negative control LV-transfected group $\left({ }^{*} \mathrm{P}<0.05\right)$. However, the ILK-siRNA-LV-transfected group exhibited a suppressed cell proliferation compared with the control group, whether or not the cells were exposed to TGF- $\beta 2\left({ }^{*} \mathrm{P}<0.05,{ }^{* *} \mathrm{P}<0.05\right)$. 
A
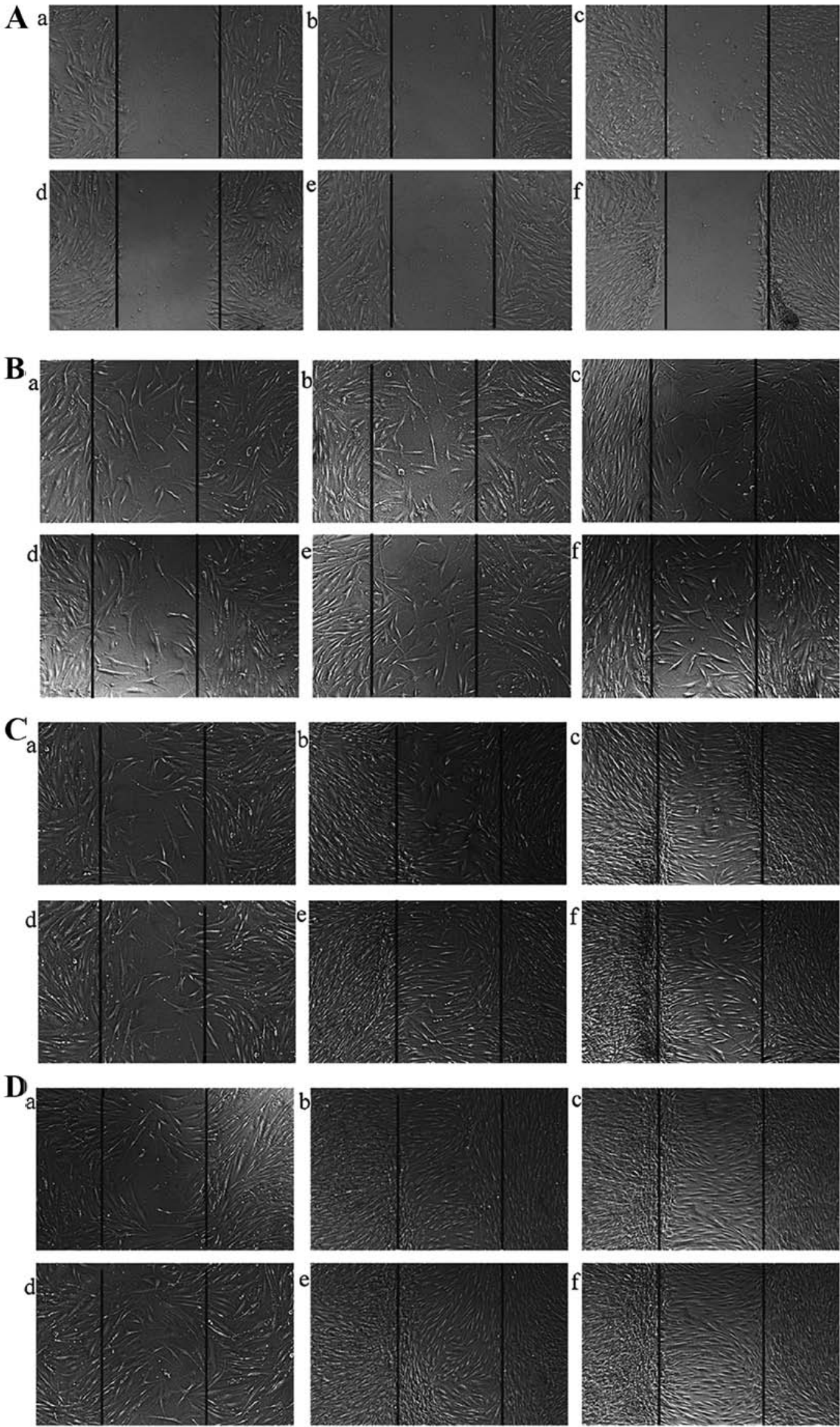

Figure 5. (A) Images (x50 magnification) of wounds made on the cell monolayer. (B) Results of wound healing assay indicated that the migration distance covered in each group at $24 \mathrm{~h}$ was similar. (C) The distance covered by cells in the experimental groups (a and d) was significantly shorter than that of the cells in the control groups (b, c, e and f) at $48 \mathrm{~h}$. (D) The wound healed in the control group at $72 \mathrm{~h}$. Panel a, integrin-linked kinase (ILK)-siRNA-LV-transfected group; panel b, negative control LV-transfected group; panel c, normal control group; panel d, ILK-siRNA-LV-transfected group exposed to transforming growth factor- $\beta 2$ (TGF- $\beta 2$ ); panel e, negative control LV-transfected group exposed to TGF- $\beta 2$; and panel f, normal control group exposed to TGF- $\beta 2$. 
A
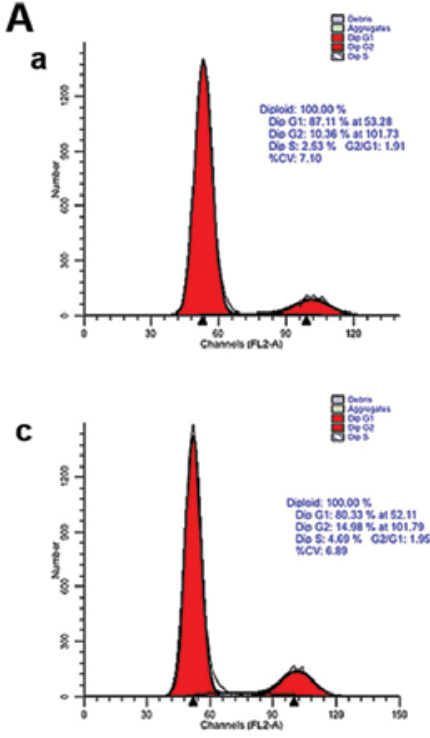

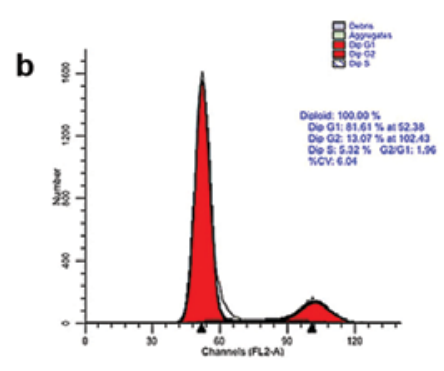

d

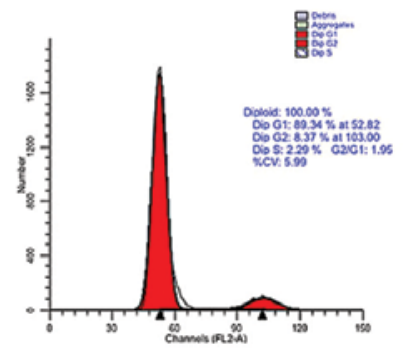

B

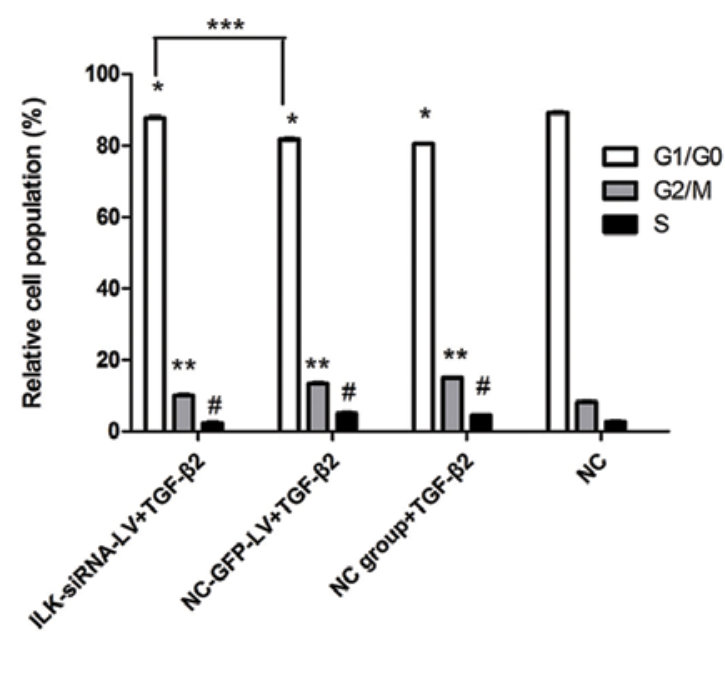

Figure 6. Cell cycle distribution detected by (A) flow cytometry and (B) results of quantification. Cells in the 3 groups (panel a, integrin-linked kinase (ILK)siRNA-LV-transfected group; panel b, NC-GFP-LV-transfected group; and panel c, NC group) were exposed to transforming growth factor- $\beta 2$ (TGF- $\beta 2$ ) for $48 \mathrm{~h}$. The cells shown in panel $\mathrm{d}$ were not exposed to TGF- $\beta 2$. There were more cells in the G1/G0 phases in the ILK-siRNA-LV-transfected group than in the NC-GFP-LV-transfected group $\left({ }^{* * *} \mathrm{P}<0.05\right)$. The percentage of cells in the G1/G0 phase decreased in the NC-GFP-LV-transfected group and in the NC group exposed to TGF- $\beta 2$ compared with the cells in the NC group not exposed to TGF- $\beta 2$ ("P $<0.05)$. The percentage of cells in the G2/M and S phases increased $\left({ }^{* *} \mathrm{P}<0.05,{ }^{*} \mathrm{P}<0.05\right)$.

not exposed to TGF- $\beta 2$ (Fig. 6B). In the presence of TGF- $\beta 2$, the percentages of cells in the G1/G0 phase in the ILK-siRNALV-transfected group significantly increased compared to the control groups (NC-GFP-LV group and NC group). These results indicate that in the presence of TGF- $\beta 2$, the silencing of ILK induces G1 phase cell cycle arrest in HTFs (Fig. 6A and B).

Effects of silencing of ILK on the protein expression of $\alpha$-SMA, cyclin DI and E-cadherin in HTFs exposed to TGF- $\beta 2$. The expression and distribution of E-cadherin and $\alpha$-SMA in the HTFs were detected by immunofluorescence staining. Under physiological conditions, E-cadherin was expressed at low levels throughout the cytoplasm (Fig. 7A, panel d) and $\alpha$-SMA was also localized in the cytoplasm in the HTFs (Fig. 7B, panel d). In the presence of TGF- $\beta 2$, in the ILK-siRNA-LVtransfected group, the staining of E-Cadherin was stronger than that in the NC-GFP-LV-transfected group and the NC group (Fig. 7A, panels a and c). Staining for $\alpha$-SMA was present diffusely and stronger in the NC-GFP-LV-transfected group and $\mathrm{NC}$ group (Fig. 7B, panels $\mathrm{b}$ and c) than in the ILK-siRNA-LV-transfected group (Fig. 7B, panel a).

The results of western blot analysis revealed that ILK was silenced at the protein level. Simultaneously, compared with the normal control group not exposed to TGF- $\beta 2$, the significant upregulation of cyclin D1 and $\alpha$-SMA expression, and the downregulation of E-cadherin expression were observed following exposure to TGF- $\beta 2$ for $48 \mathrm{~h}$ (Fig. 8). These results indicated that TGF- $\beta 2$ stimulated the differentiation capacity of HTFs. This capacity was suppressed when ILK was silenced. The expression of $\alpha$-SMA and cyclin D1 decreased in the ILK-siRNA-LV-transfected group compared with the NC-GFP-LV-transfected group, while E-Cadherin expression increased (Fig. 8).

\section{Discussion}

Glaucoma filtration surgery involves the surgical formation of an artificial drainage pathway from the anterior chamber to the subconjunctival space. The rate of fluid drainage from the subconjunctival space is actually the determining factor in the reduction of IOP (19). Though there are many new devices for glaucoma filtration surgery, scarring is the main cause of surgical failure $(20,21)$. HTFs play a major role in this process. The culture, isolation and expansion of HTFs has been demonstrated (22). The tissue explant technique is the most common method used. In this study, we successfully cultured HTFs at a low cost, which is a good basis for studying post-operative scarring. The cultured cells were in a fibrocyte-like form and had a spindle-shape, and were arranged in a fasciculus or whirlpool pattern after using the tissue explants adherence method. The cells were identified by cytoplasmic proteins. Vimentin, a type of intermediate filament, is a specific marker protein of mesenchymal cells, while keratin makes up the largest subgroup of intermediate filament proteins and represents the most abundant protein in epithelial cells (23). As HTFs are a type of mesenchymal cells, the results of immunofluorescence staining, and those of the morphology and growth of the cells proved that the cells we cultured were fibroblasts.

TGF- $\beta$ governs developmental processes and regulates homeostasis by controlling cellular proliferation, survival, differentiation and migration (24). TGF- $\beta$ is produced by platelets, the vascular endothelium and macrophages in the wound healing process. It is a hot spot of research in cancer, and in cardiovascular, musculoskeletal or fibrotic diseases $(25,26)$. Due to its significant effect on fibrosis, TGF- $\beta$ has become an antiscarring target in ocular disease research (27). For example, in an animal experiments, in a mouse corneoscleral wound model, fibroblasts developed within the first 2 days of surgery, the 
A

E-Cadherin

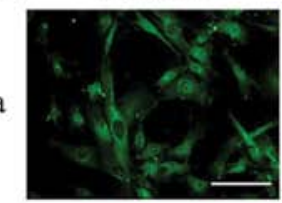

b
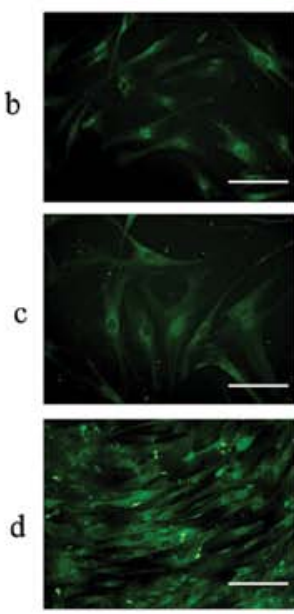

DAPI
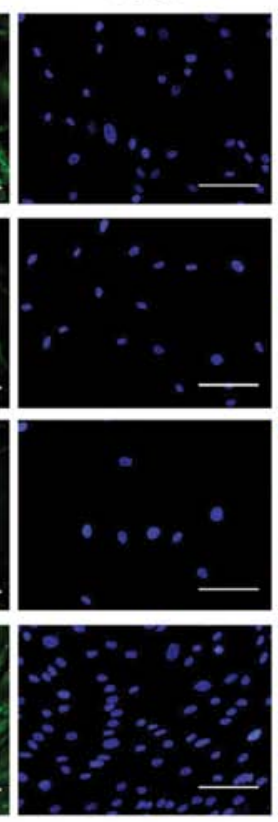

Merge
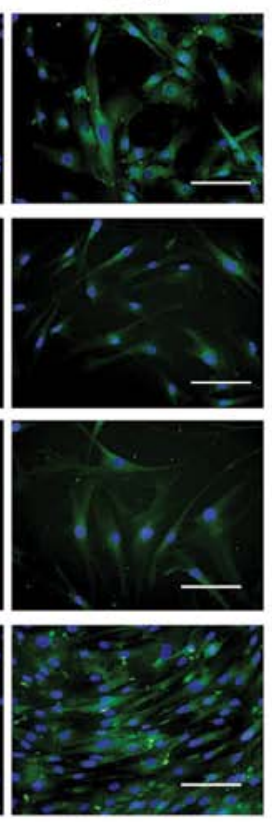

B
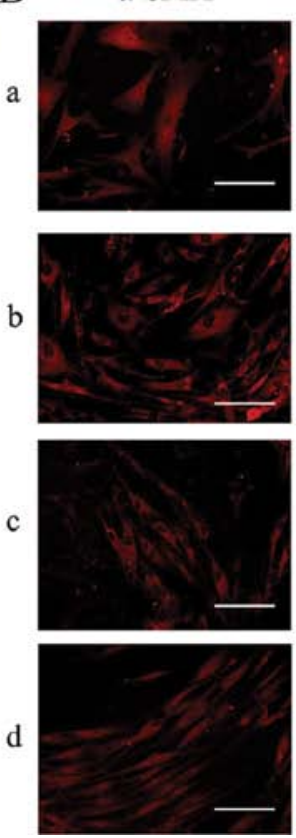

DAPI
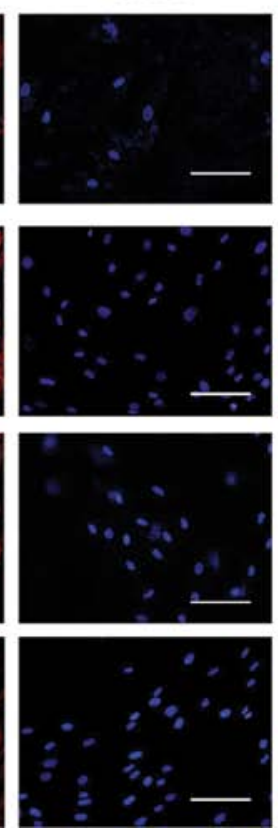

Merge
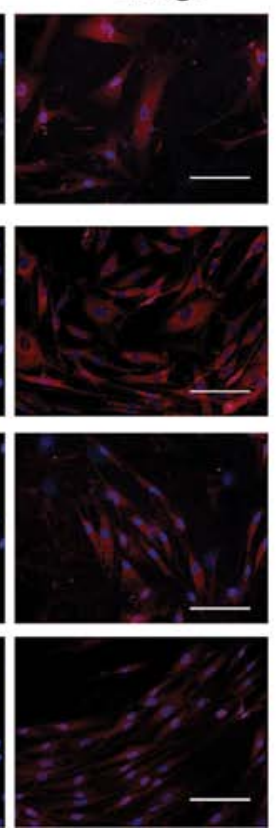

Figure 7. Images (x200 magnification) of immunofluorescence staining of (A) E-cadherin (green) and (B) $\alpha$-smooth muscle actin ( $\alpha$-SMA) (red). Panel a, integrin-linked kinase (ILK)-siRNA-LV-transfected group exposed to transforming growth factor- $\beta 2$ (TGF- $\beta 2$ ); panel b, NC-GFP-LV-transfected group exposed to TGF- $\beta 2$; panel c, normal control group exposed to TGF- $\beta 2$; and panel d, normal control group not exposed to TGF- $\beta 2$. Scale bars, $100 \mu \mathrm{m}$.

collagen component increased over time and TGF- $\beta 2$ appeared early in the process at high levels (28). In our study, TGF- $\beta 2$ was reconfirmed to stimulate the proliferation of HTFs by increasing the percentages of cells in the G2/M and S phases of the cell cycle. However, we did not observe any obvious promoting effects of TGF- $\beta 2$ on cell proliferation and migration. This may be due to the fact that HTFs have a strong self-renewal ability, multi-differentiation potential, and a strong proliferative and migration ability in vitro. During the process of cultivating, cells trigger the stress response to external environments, which even causes differentiation into myofibroblasts to some degree.

ILK is overexpressed in many types of human diseases downstream of the TGF- $\beta$ signaling pathway. At present, research on ILK is mainly focused on invasion and metastasis of epithelial malignancies or on epithelial to mesenchymal transition (EMT) in tissue fibrosis. For example, in a study on lung fibrosis, TGF- $\beta 1$ was used to induce fibrotic characteristics in alveolar epithelial cells. ILK was found to be involved in the upregulation of vimentin (29). In a study on peritoneal fibrosis, a low vimentin expression and a high E-cadherin expression was observed when ILK gene expression was silenced by siRNA, suggesting that EMT, which is a process for fully differentiated epithelial cells to undergo a phenotypic change into fibroblasts via diverse intracellular signaling pathways, was inhibited (30). Another study also demonstrated that the migratory and invasive ability of SW480 cells was significantly enhanced following the enforced overexpression of ILK. SW480 cells stably overexpressing ILK underwent EMT, with a decreased expression of E-cadherin, and an increased expression of vimentin, Snail and Slug (16). In addition, ILK overexpression is associated with tumor progression. In a previous study, both ILK mRNA and protein expression levels were significantly upregulated in primary colorectal cancer samples compared with their corresponding normal tissues (31).
Another study demonstrated that when TGF- $\beta 1$ was used to promote EMT and migration in mammary epithelial cells, the TGF- $\beta 1$-induced processes were significantly suppressed by inhibiting ILK activity. ILK was essential in TGF- $\beta 1$-induced EMT in mammary epithelial cells (32). ILK silencing by siRNA has been shown to inhibit EMT, and the metastasis and growth of bladder cancer cells and tongue cancer cells (33-35). Moreover, as previously demonstrated, the phosphorylation of ILK can lead to the phosphorylation of Akt and the inactivation of ILK reverses the $\alpha$-parvin (PARVA)-induced invasion of lung cancer cells (36). In ocular disease research, ILK is required for the complete activation of MAPK and PI3K/Akt signaling, downstream of the FGF receptor in lens epithelial cells during development, and is involved in epithelial proliferation, survival and subsequent fibre differentiation (37). In addition to the studies at the cellular level, some experiments on animals have suggested that cardiac-specific ILK knockout mice spontaneously developed lethal dilated cardiomyopathy and heart failure with an early increase in apoptosis, fibrosis, and cardiac inflammation (38). Nevertheless, the effect of ILK in HTFs which is mesenchymal origin cell has not been reported. In this study, we found that the silencing of ILK suppressed the proliferation and migration of HTFs induced by TGF- $\beta 2$, along with G1 phase cell cycle arrest. The downregulation of cyclin D1 expression was consistent with this phenomenon.

Under physiological conditions, fibroblasts exist in the interstitium, maintain the dynamic balance of the interstitium and adjacent tissue, which plays an important role on cell proliferation and extracellular matrix production. Many factors can stimulate fibroblast activation and differentiation into myofibroblasts that exhibit a stronger ability for proliferation and secrete much more extracellular matrix. Myofibroblasts are the major source of extracellular matrix during fibrosis. More 


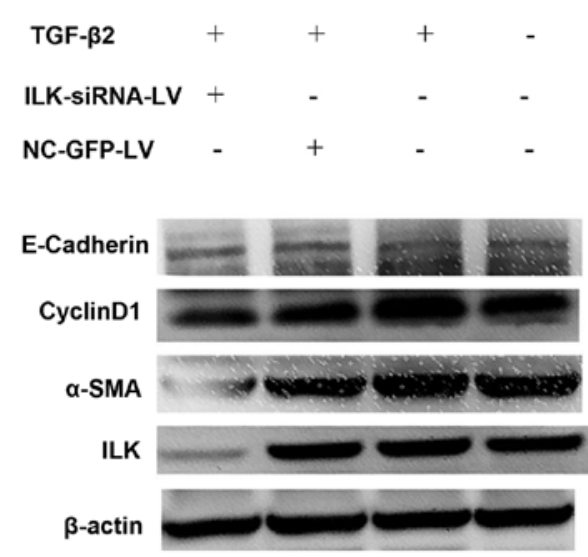

CyclinD1

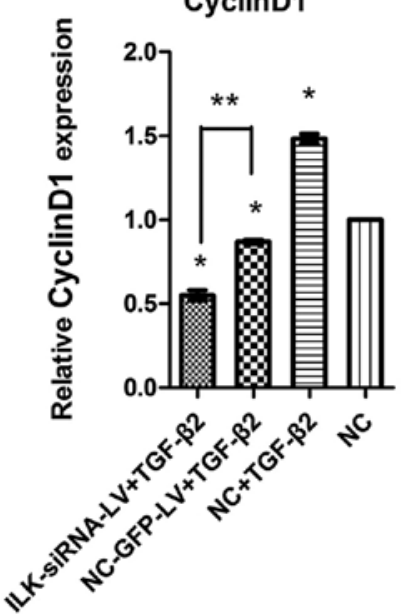

a-SMA

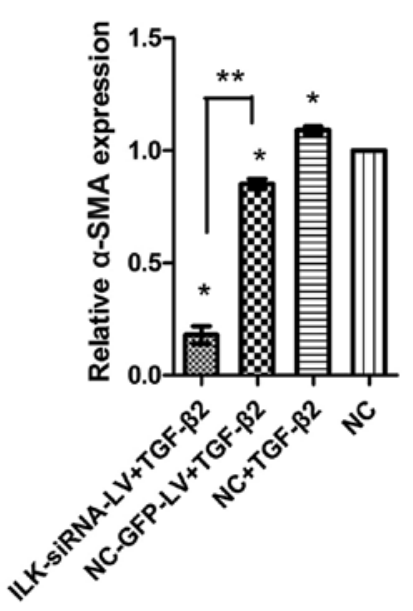

E-cadherin

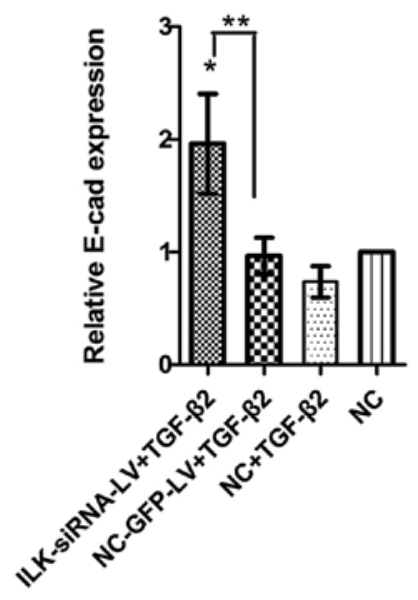

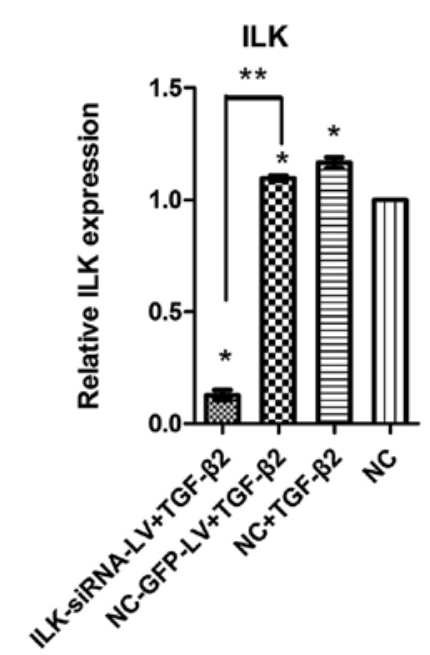

Figure 8. Results of western blot analysis showin the effects of silencing integrin-linked kinase (ILK) on the protein expression of $\alpha$-smooth muscle actin ( $\alpha$-SMA), cyclin D1 and E-cadherin in transforming growth factor- $\beta 2$ (TGF- $\beta 2$ )-exposed human Tenon's capsule fibroblasts (HTFs). ${ }^{*} \mathrm{P}<0.05$ compared with normal control group, ${ }^{* * *} \mathrm{P}<0.05$ compared with NC-GFP-LV group.

importantly, myofibroblasts have the ability to contract as smooth muscle cells (39-41). In this study, the expression of $\alpha$-SMA, as a marker of myofibroblasts, was decreased in the cells in which ILK was silenced, which indicated that the cell differentiation ability was decreased. However, $\alpha$-SMA was observed in both the TGF- $\beta 2$-exposed group and the normal control group in our study. We hypothesized that this may be due to the fact that some HTFs had already differentiated into myofibroblasts prior to exposure to TGF- $\beta 2$. E-cadherin is a subclass of the cadherin family that plays a major role in cell-cell adhesion in the normal epithelium. E-cadherin is located on cell-cell boundaries in the normal epithelium (42). It has been reported that the reduction or loss of E-cadherin expression is associated with invasion and metastasis in cancer. Changes in E-cadherin immunoreactivity and cellular localization occur in the premalignant metaplastic epithelium of the oesophagus (43). Cadherins also play an integral role in neuronal morphogenesis (44). The roles of E-cadherin in different types of cells remain to be fully elucidated. In this study, we found that E-cadherin was expressed in the cytoplasm in HTFs, which is not localized in the cell membrane as in epithelial cells. This result indicates that E-cadherin may not act as an intercellular adhesion molecule, but as a regulatory factor of cell activity in HTFs.
In conclusion, by transfecting HTFs with ILK-siRNA-LV to silence ILK expression, we found that the silencing of ILK attenuated the abnormal proliferation of fibroblasts induced by TGF- $\beta 2$ and the migration ability also decreased after ILK gene silencing. ILK may be an important factor in scarring following glaucoma filtration surgery and the downstream factor of the TGF- $\beta$ signaling pathway. Our study demonstrated that ILK silencing also suppressed the differentiation ability of HTFs induced by TGF- $\beta 2$. However, several limitations to our study need to be pointed out. We only observed the biological behavior of HTFs, following ILK gene silencing, and the molecular mechanisms responsible for this process were not revealed. In further studies, we aim to to examine the effects of the silencing of ILK on other signal transduction pathways (Smads, Rock, PI3K), in order to fully explore the complex interaction among these pathways. This may provide a new direction for improving the success rate of glaucoma filtration surgery.

\section{Acknowledgements}

This study was supported by National Natural Science Foundation of China (NSFC; no. 81300765). 


\section{References}

1. Quigley HA and Broman AT: The number of people with glaucoma worldwide in 2010 and 2020. Br J Ophthalmol 90: 262-267, 2006

2. Grisanti S, Szurman P, Warga M, Kaczmarek R, Ziemssen F Tatar O and Bartz-Schmidt KU: Decorin modulates wound healing in experimental glaucoma filtration surgery: a pilot study. Invest Ophthalmol Vis Sci 46: 191-196, 2005.

3. Ashaye AO and Komolafe OO: Post-operative complication of trabeculectomy in Ibadan, Nigeria: outcome of 1-year follow-up. Eye (Lond) 23: 448-452, 2009.

4. Cordeiro MF: Role of transforming growth factor beta in conjunctival scarring. Clin Sci (Lond) 104: 181-187, 2013.

5. Denk PO, Hoppe J, Hoppe V and Knorr M: Effect of growth factors on the activation of human Tenon's capsule fibroblasts. Curr Eye Res 27: 35-44, 2003.

6. Meyer-Ter-Vehn T, Katzenberger B, Han H, Grehn F and Schlunck G: Lovastatin inhibits TGF-beta-induced myofibroblast transdifferentiation in human tenon fibroblasts. Invest Ophthalmol Vis Sci 49: 3955-3960, 2008.

7. Miyazono K: TGF-beta signaling by Smad proteins. Cytokine Growth Factor Rev 11: 15-22, 2000.

8. Piek E, Ju WJ, Heyer J, Escalante-Alcalde D, Stewart CL, Weinstein M, Deng C, Kucherlapati R, Bottinger EP and Roberts AB: Functional characterization of transforming growth factor beta signaling in Smad2- and Smad3-deficient fibroblasts. J Biol Chem 276: 19945-19953, 2001.

9. Grotendorst GR, Rahmanie H and Duncan MR: Combinatorial signaling pathways determine fibroblast proliferation and myofibroblast differentiation. FASEB J 18: 469-479, 2004.

10. Schiller M, Javelaud D and Mauviel A: TGF-beta-induced SMAD signaling and gene regulation: consequences for extracellular matrix remodeling and wound healing. J Dermatol Sci 35: 83-92, 2004.

11. Wu C and Dedhar S: Integrin-linked kinase (ILK) and its interactors: a new paradigm for the coupling of extracellular matrix to actin cytoskeleton and signaling complexes. J Cell Biol 155 : 505-510, 2001.

12. Li J, Yang ZL, Ren X, Zou Q, Yuan Y, Liang L, Chen M and Chen S: ILK and PRDX1 are prognostic markers in squamous cell/adenosquamous carcinomas and adenocarcinoma of gallbladder. Tumour Biol 34: 359-68, 2013.

13. Li Y, Yang J, Dai C, Wu C and Liu Y: Role for integrin-linked kinase in mediating tubular epithelial to mesenchymal transition and renal interstitial fibrogenesis. J Clin Invest 112: 503-516, 2003.

14. Vi L, de Lasa C, DiGuglielmo GM and Dagnino L: Integrin-linked kinase is required for TGF- $\beta 1$ induction of dermal myofibroblast differentiation. J Invest Dermatol 131: 586-593, 2011.

15. Bravou V, Klironomos G, Papadaki E, Taraviras S and Varakis J: ILK over-expression in human colon cancer progression correlates with activation of beta-catenin, down-regulation of E-cadherin and activation of the Akt-FKHR pathway. J Pathol 208: 91-99, 2006.

16. Liang F, Zhang S, Wang B, Qiu J and Wang Y: Overexpression of integrin-linked kinase (ILK) promotes glioma cell invasion and migration and down-regulates E-cadherin via the $N F-\kappa B$ pathway. J Mol Histol 45: 141-151, 2014

17. Persad S, Attwell S, Gray V, Delcommenne M, Troussard A, Sanghera J and Dedhar S: Inhibition of integrin-linked kinase (ILK) suppresses activation of protein kinase B/Akt and induces cell cycle arrest and apoptosis of PTEN-mutant prostate cancer cells. Proc Natl Acad Sci U S A 97: 3207-12, 2000.

18. Verreault M and Bally MB: siRNA-mediated integrin-linked kinase suppression: nonspecific effects of siRNA/cationic liposome complexes trigger changes in the expression of phosphorylated-AKT and mTOR independently of ILK silencing. Oligonucleotides 19: 129-140, 2009.

19. Yu DY, Morgan WH, Sun X, Su EN, Cringle SJ, Yu PK, House P, Guo W and Yu X: The critical role of the conjunctiva in glaucoma filtration surgery. Prog Retin Eye Res 28: 303-328, 2009.

20. Holló G: Wound healing and glaucoma surgery: modulating the scarring process with conventional antimetabolites and new molecules. Dev Ophthalmol 50: 79-89, 2012.

21. Yan ZC, Bai YJ, Tian Z, Hu HY, You XH, Lin JX, Liu SR, Zhuo YH and Luo RJ: Anti-proliferation effects of Sirolimus sustained delivery film in rabbit glaucoma filtration surgery. Mol Vis 17: 2495-2506, 2011.

22. De Falco E, Scafetta G, Napoletano C, Puca R, Vingolo EM, Ragona G, Iorio O and Frati G: A standardized laboratory and surgical method for in vitro culture isolation and expansion of primary human Tenon's fibroblasts. Cell Tissue Bank 14: 277-287, 2013.
23. Stahnke T, Löbler M, Kastner C, Stachs O, Wree A, Sternberg K, Schmitz KP and Guthoff R: Different fibroblast subpopulations of the eye: a therapeutic target to prevent postoperative fibrosis in glaucoma therapy. Exp Eye Res 100: 88-97, 2012.

24. Horbelt D, Denkis A and Knaus P: A portrait of transforming growth factor $\beta$ superfamily signalling: background matters. Int J Biochem Cell Biol 44: 469-474, 2012.

25. Karon P, Olejek A and Olszak-Wasik K: TGF- $\beta$ expression in vulvar cancer. Ginekol Pol 85: 847-851, 2014

26. Bentley-Hewitt KL, De Guzman CE, Ansell J, Mandimika T, Narbad A and Lund EK: Polyunsaturated fatty acids modify expression of TGF- $\beta$ in a co-culture model ultilising human colorectal cells and human peripheral blood mononuclear cells exposed to Lactobacillus gasseri, Escherichia coli and Staphylococcus aureus. Eur J Lipid Sci Technol 116: 505-513, 2014

27. Zhu X, Li L, Zou L, Zhu X, Xian G, Li H, Tan Y and Xie L: A novel aptamer targeting TGF- $\beta$ receptor II inhibits transdifferentiation of human tenon's fibroblasts into myofibroblast. Invest Ophthalmol Vis Sci 53: 6897-6903, 2012.

28. Mietz H, Chévez-Barrios P and Lieberman MW: A mouse model to study the wound healing response following filtration surgery. Graefes Arch Clin Exp Ophthalmol 236: 467-475, 1998

29. Kavvadas P, Kypreou KP, Protopapadakis E, Prodromidi E, Sideras $P$ and Charonis AS: Integrin-linked kinase (ILK) in pulmonary fibrosis. Virchows Archiv 457: 563-75, 2010

30. Luo L, Liu H, Dong Z, Sun L, Peng Y and Liu F: Small interfering RNA targeting ILK inhibits EMT in human peritoneal mesothelial cells through phosphorylation of GSK $3 \beta$. Mol Med Rep 10: 137-144, 2014.

31. Li R, Liu B, Yin H, Sun W, Yin J and Su Q: Overexpression of integrin-linked kinase (ILK) is associated with tumor progression and an unfavorable prognosis in patients with colorectal cancer. J Mol Histol 44: 183-189, 2013.

32. Serrano I, McDonald PC, Lock FE and Dedhar S: Role of the integrin-linked kinase (ILK)/Rictor complex in TGF $\beta$-1-induced epithelial-mesenchymal transition (EMT). Oncogene 32: 50-60, 2013.

33. Yao X, Li D, Xiong DM, Li L, Jiang R and Chen JX: A novel role of ribonuclease inhibitor in regulation of epithelial-to-mesenchymal transition and ILK signaling pathway in bladder cancer cells. Cell Tissue Res 353: 409-423, 2013

34. Li L, Pan XY, Shu J, Jiang R, Zhou YJ and Chen JX: Ribonuclease inhibitor up-regulation inhibits the growth and induces apoptosis in murine melanoma cells through repression of angiogenin and ILK/PI3K/AKT signaling pathway. Biochimie 103: 89-100, 2014

35. Xing Y, Qi J, Deng S, Wang C, Zhang L and Chen J: Small interfering RNA targeting ILK inhibits metastasis in human tongue cancer cells through repression of epithelial-to-mesenchymal transition. Exp Cell Res 319: 2058-2072, 2013.

36. Huang AH, Pan SH, Chang WH, Hong QS, Chen JJ and Yu SL: PARVA promotes metastasis by modulating ILK signalling pathway in lung adenocarcinoma. PLoS One 10: e0118530, 2015.

37. Teo ZL, McQueen-Miscamble L, Turner K, Martinez G, Madakashira B, Dedhar S, Robinson ML and de Iongh RU: Integrin linked kinase (ILK) is required for lens epithelial cell survival, proliferation and differentiation. Exp Eye Res 121: 130-142, 2014.

38. Dai J, Matsui T, Abel ED, Dedhar S, Gerszten RE, Seidman CE, Seidman JG and Rosenzweig A: Deep sequence analysis of gene expression identifies osteopontin as a downstream effector of integrin-linked kinase (ILK) in cardiac-specific ILK knockout mice. Circ Heart Fail 7: 184-193, 2014

39. Buntrock P: Ultrastructural characteristics of fibroblasts, myofibroblasts, and fibroclasts in the process of wound healing (author's transl). Zentralbl Allg Pathol 124: 48-59, 1980 (In German).

40. Ohtani H and Sasano N: Stromal cell changes in human colorectal adenomas and carcinomas. An ultrastructural study of fibroblasts, myofibroblasts, and smooth muscle cells. Virchows Arch A Pathol Anat Histopathol 401: 209-222, 1983.

41. Desmoulière A, Geinoz A, Gabbiani F and Gabbiani G: Transforming growth factor-beta 1 induces alpha-smooth muscle actin expression in granulation tissue myofibroblasts and in quiescent and growing cultured fibroblasts. J Cell Biol 122: 103-111, 1993.

42. Oka H, Shiozaki H, Kobayashi K, Inoue M, Tahara H, Kobayashi T, Takatsuka Y, Matsuyoshi N, Hirano S, Takeichi M, et al: Expression of E-cadherin cell adhesion molecules in human breast cancer tissues and its relationship to metastasis. Cancer Res 53: 1696-16701, 1993.

43. Jankowski J, Newham P, Kandemir O, Hirano S, Takeichi M and Pignatelli M: Differential expression of e-cadherin in normal, metaplastic and dysplastic esophageal mucosa - a putative biomarker. Int J Oncol 4: 441-448, 1994.

44. Suzuki SC and Takeichi M: Cadherins in neuronal morphogenesis and function. Dev Growth Differ 50 (Suppl 1): S119-S130, 2008 